Original Article

\title{
The evolutionary complexity of social and economic systems: The inevitability of uncertainty and surprise
}

\author{
Peter M. Allen*, Liz Varga and Mark Strathern \\ Complex Systems Research Centre, Cranfield University, Cranfield MK43 OAL, UK. \\ *Corresponding author.
}

\begin{abstract}
Social systems are evolving, multi-scale, spatio-temporal structures with emergent functions, needs and capabilities. One sensible definition of an 'extreme' event would be one that led to some qualitative, structural change in the system, as it went beyond the current 'control limits' of the system. Indeed, we can view any current structure and organization that characterize a system as being forged out of the past events and crises that led to change and innovation. Each individual, group, firm, corporation, shareholder and observer experience 'pathdependent learning', whereby current learning is conditional by the events and decisions taken in the past. In reality, the 'new' system or structure that comes into being at a given time, although initially seen as a successful response to some past problem, will move into an unknowable future that will necessarily end in some new crisis, the emergence of some unexpected new factors, effects or implications that will have to be dealt with. A simple probabilistic model of event dynamics is presented showing that the discussions of power laws and self-organized criticality assume a stationary situation and the probability of given events. We contrast this with examples from human systems that are about qualitative, structural change and path-dependent learning. The first example involves the handling of severe motorway traffic accidents and the second describes the evolution of supply chains by trial and error, as new practices are adopted or rejected in a changing competitive economic environment. Social and economic systems must evolve, and will do so through the occurrence of seemingly 'extreme' events, requiring adaptation and change. The important lesson therefore is to accept that there will be always a potential for unexpected and surprising events and that we need to build systems that are robust and resilient to their occurrence.
\end{abstract}

Risk Management (2010) 12, 9-30. doi:10.1057/rm.2009.15

Keywords: complexity; power laws; evolution; instability 


\section{Introduction}

The key factor in our understanding of evolved and evolving systems is that over time they have undergone successive structural instabilities that have led to the current multi-level structures of elements and links that constitute our system. The course of rivers is not explained by the current average flows but the history of past extreme events, when particular storms shifted the bed to a new path. So, landscapes, ecologies, towns, cities and organizations have all been shaped by the extreme events that have marked their past, when the previous structures proved to be inadequate to deal with what was happening. Either the system was overwhelmed and therefore disappeared or became extinct, or it managed to adapt and respond so that its repertoire included behaviours that dealt successfully with that particular threat. Indeed, what becomes evident is that we inhabit a world of evolved structures and organizations that are the fruit of such an adaptive evolutionary process, and we may almost say that the only things that have really mattered are the 'extreme' events that provoked change in the system. Evolved entities are really created both by the structures that emerged and by the extreme events that provoked their adaptive responses and particularities.

This now changes the view that is usually taken when writing about 'extreme events', where usually the idea is how to protect the system from earthquake shock, sudden flood, extreme heat wave or network failure. It is an idea of preservation, of defence and of maintaining and restoring functionalities. However, looked at in the broader perspective of evolution, we see that in order to fit into a changing world, extreme events are inevitable and are really the challenges through which progress has occurred. Evolution selects for those that can evolve and respond, and not for any particular behaviour or characteristic. Success in the past is no guarantee of its continuation and it is perhaps not wholly irrelevant to point to the fact that it was the extreme event that wiped out the dinosaurs that allowed humans to colonize the Earth.

The ever present danger is to allow a system existing under recent, benevolent conditions to be optimized and modified in a way that relies upon the continuation of these benevolent conditions. This is about the idea of 'hidden assumptions' where, although initially people may be aware of the various factors they are relying on, gradually this knowledge can erode, and so it comes as a massive 'surprise' when an assumption fails and a crisis is triggered. Time scales of processes of construction and development need to take into account those of various possible events that might threaten them. So riverside dwellings will probably heed the effects of annual and 10-year events, but might fail to consider those that only occur on average every 100 or 200 years. But in a changing world, these frequencies are not fixed and so we need to try to explore the dynamics of our environment and modify our decisions accordingly. 


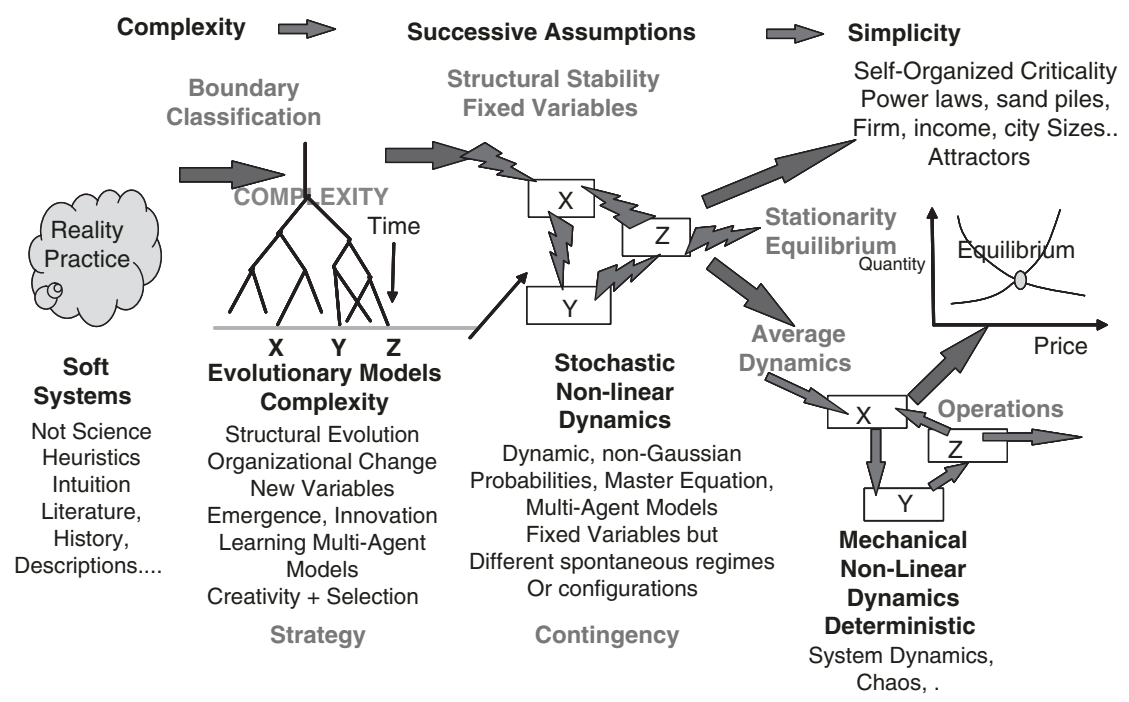

Figure 1: The overall conceptual scheme of increasingly simplified representations obtained by using successive additional assumptions.

In several previous papers (Allen, 2001a, b), it was shown how social and economic systems are described by co-evolutionary, complex systems models in which both the agents, the structure of their interactions and the products and services that they exchange evolve qualitatively.

In the short term, if this can be defined, a systems model of the situation can be useful for operational matters, although in reality the particular system of the moment will actually evolve onwards, and in that case may destroy both current understanding and functionalities in a seeming crisis. This may be studied to a degree with a slightly longer-term 'contingency' model that allows for the possible effects of changes and fluctuations in the environment of the system. However, in the longer term nothing can avoid the fact that 'systems' evolve and co-evolve qualitatively over time, as events occur that change them, creating new entities and components, and new problems and opportunities. Such events are clearly 'extreme', in that they change the system irreversibly, but they can be seen as both good and bad, as they may either result in the destruction of some elements or the creation or discovery of some new ones. This overall conceptual scheme is shown in Figure 1.

These new ideas of complex systems encompass evolutionary processes in general, and apply to the social, cultural, economic, technological, psychological and philosophical aspects of our realities. What matters over time is the expansion of any system into new dimensions and conceptual spaces, as a result of successive instabilities involving dimensions additional to those the current 'system' appears to occupy. In a way, the 'extreme' events of creation 
and destruction are really the most significant factors, as their accumulation is what actually builds the evolved system. A river basin is not changed by the average flows of a given period, but by the exceptional ones that change its course. So complexity ideas embrace the idea of 'path dependency' and mean that everything that matters is the nature of the crises and innovations that actually occur.

This idea of evolution as a question of 'invadability', with respect to what was not yet in the system, was the subject of a very early paper by the author (Allen, 1976). Essentially then, systems are seen as temporary, emergent structures that result from the self-reinforcing non-linear interactions that result from successive 'invasions'. History is written not only by some process of 'rational improvement' in its internal structure but more fundamentally by its dialogue with elements that are not yet in the system - successive experimental linkages that either are rejected by the system, or which 'take off' and modify the system irreversibly.

Rational improvement of internal structure, the traditional domain of 'systems' thinking', supposes that the system has a purpose, and known measures of 'performance' that can indicate the direction of improvements. But, this more fundamental structural evolution of complex systems that results from successive invasions of the system by new elements and entities is characterized by emergent properties and effects that lead to new attributes, purposes and performance measures. In the next section, however, we shall consider the mathematics of 'events' where it is assumed that they are of a fixed kind and are described by a fixed probability distribution. Such a view is not evolutionary, as it supposes a fixed probability distribution.

\section{Extreme Events and Qualitative Change}

The words 'extreme events' often imply that there is some fixed probability distribution of possible events that go from the very frequent, through the less frequent to the very infrequent and extreme. On the whole, the words are also associated with negative events - earthquakes, storms, and so on, but as we shall argue later, if we define extreme events as those that change the system qualitatively, then these are in fact the motor of evolution and adaptation, and should not be seen as negative. In addition, in reality an 'event' should be something that is not part of the normal processes of the system, but much of the literature on 'extreme events' is simply about predicting the frequency of a particular, known type of 'event' that might be an earthquake but is often also firm or city size, where extreme is taken to mean very large. The simple probabilistic maths supposes a probability of individual growth or decline in a population of essentially similar objects, leading to a fixed size distribution as a result. But we want to underline the fact that the real reasons that lie 
behind the evolution of human systems is about qualitative differences between individual elements, resulting from new capabilities, structural organization or simply new ideas and innovations. In this view, instead of 'extreme events' referring to the occurrence of a known, given phenomenon, we see them as those events that change the system or situation qualitatively - as evolutionary steps.

In order to clarify much of the discussion on extreme events, let us consider a model of the distribution of 'events' of the same kind but of different size by a set of equations that link the dynamics of the events $x(i)$, with their size. An event might be the spread of a new virus, the probability of a 'storm' of a given strength, or the probability of the Richter scale strength of an earthquake. So $i$ may range from 1 to, say, 50 for example. Events begin and then over time either grow or shrink. An extreme event would be one that grew to a very large size before disappearing. The 'event dynamics' can be generated from the following equations:

$$
\begin{aligned}
& \mathrm{d} x(1)=f i \cdot(1-\text { TotalX } / N)-m(1) \cdot x(1)-x(1) / \tau+x(2) / \tau \\
& \text { For } i=2 \text { to MaxSize }-1: \\
& \mathrm{d} x(i)=x(i-1) / \tau-m(i) \cdot x(i)-x(i) / \tau+x(i+1) / \tau-x(i) / \tau \\
& \text { For } x=\text { MaxSize: } \\
& \mathrm{d} x(\text { MaxSize })=x(\text { MaxSize }-1) / \tau-m(\text { MaxSize }) \cdot x(\text { MaxSize })-x(\text { MaxSize }) / \tau
\end{aligned}
$$

Where Total $X$ is the sum of the events $x$ present. $N$ is a limiting factor. MaxSize is the maximum size category considered. $\tau$ is the time for an event to grow from one size to the next and $m(i)$ is the rate of failure at size $i$. If we assume, as we do here, that the $m(i)$ are all the same, then we are assuming size independence of the probability of decrease of an event. The equations describe how an initial event will either persist and grow or will decline and disappear. The key parameters are therefore the rate of growth $(1 / \tau)$ and the probability of decline $m$.

These equations allow us to model the changing distribution of the events of different sizes. Initial events all start with small size, and then may either fail immediately or grow to the next size. This process occurs for all sizes, if an event survives for a time $\tau$ then it will, on average, grow up to the next size above. These size classes are, of course, exponential in scale, since by using equal time steps for the passage from one level to another, we are using a 'doubling' time, arising from percentage growth rather than an absolute value.

The equations above can be run, and generate 'rank-size' rules for the largest 100 or 500 of the events. The frequency of size (probability density) is a decreasing function of size $\sim$ which could be exponential ( - alpha) or size ${ }^{\text {alpha }}$, as is shown in Figure 2. 

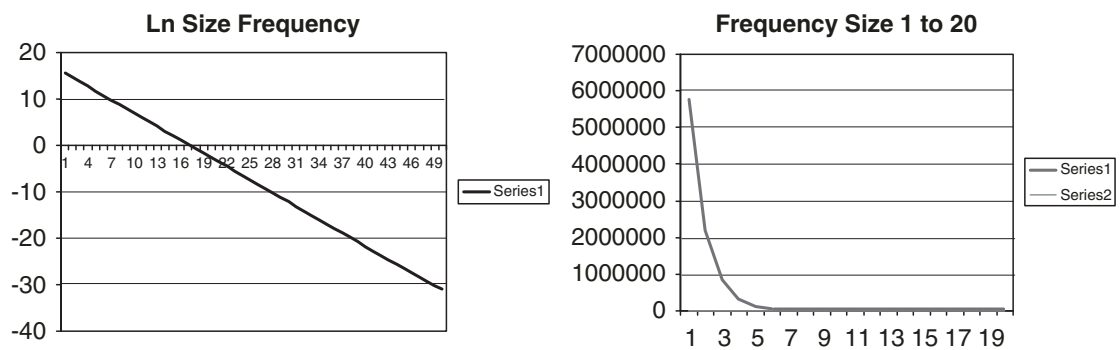

Figure 2: The model produces an exponential frequency distribution. Alpha $=-0.9295$

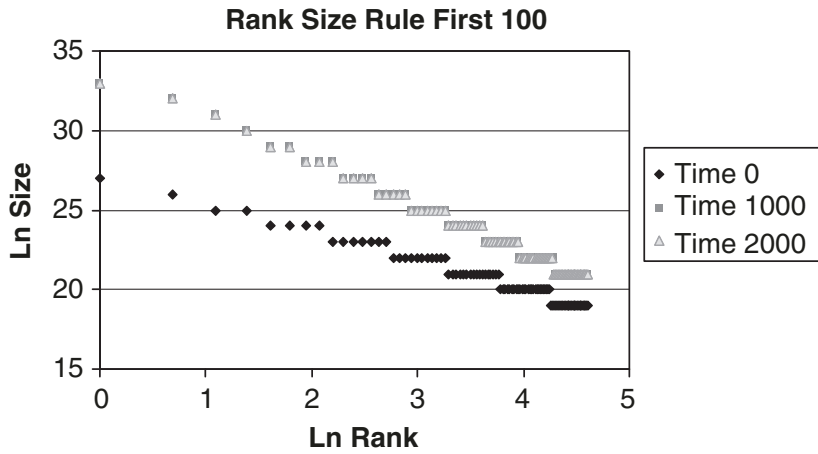

Figure 3: The rank-size rule generated by running the equations above and using the power law relations.

All the largest elements are squeezed in the probability frequency between size 17 and size 11 . Clearly, for the vanishingly small probability density this must mean that the elements ranked 1 and 2, 3 and so on must be separated by some considerable size difference, or the probability density would not fit. The shape of the curve depends on the mechanisms and parameters in the growth equations, but whatever the details of this are, the rank-size rule starting with the largest size element is unaffected by them as shown in Figure 3. But, in the simulation, the top 100 elements is a tiny fraction of the total number, which is many thousands. The linear nature of the relationship does not hold for the very small sizes, and indeed, it is here that 'strategy' and evolutionary advantage may be important.

The corresponding size of successive events is shown in Table 1 , and this is precisely the type of table that is found for things such as city- or firm-size distributions.

When we observe a power law distribution of events, then it appears to mean that there is no natural or 'preferred' size, and therefore no important economies of scale or scope, and no learning by experience. This very simple 
Table 1: The relative size of the top ranking elements of a system defined by the equations above

\begin{tabular}{lcr}
\hline Event & Ln Size & Size \\
\hline Rank 1 & 9.21 & 10000 \\
Rank2 & 8.46 & 4712 \\
Rank 3 & 8.02 & 3034 \\
Rank 4 & 7.71 & 2220 \\
Rank 5 & 7.46 & 1742 \\
Rank 6 & 7.26 & 1429 \\
Rank 7 & 7.10 & 1209 \\
Rank 8 & 6.95 & 1046 \\
Rank 9 & 6.82 & 920 \\
Rank 10 & 6.71 & 821 \\
\hline
\end{tabular}

model treats the events as 'random', without any individual features or characteristics that increase its chance of growth or decay, and since these are absent then there is no question of there being any size-dependent behaviour - such as an optimal or critical size. This is an important point because a large part of management research may be devoted to discovering the 'indicators' that will predict the different successes to be expected from new companies. Similarly, when handling a negative event such as an earthquake or a flood, research may spend much effort in trying to find the predictors of the expected size of events, in order to put appropriate levels of emergency service into operation as soon as possible.

Instead of this essentially stationary view, evolution is really built up from the novel events - innovations and mutants - that are 'retained' by the system. Instead of individuals, firms, cities or disturbance entering the system with essentially the same average parameters $(m$ and $1 / \tau)$ the invaders may have a spread of inherent performance, corresponding to some internal novelty or difference. What is the probability of survival or extinction of novel entrants with diverse $m$ and $1 / \tau$ ? The mathematics of this problem was dealt with some time ago (Allen and Ebeling, 1983) whereby the probabilistic dynamics of the growth or decay of a mutant within a population was treated. The results of this work showed that novel behaviours that were $\delta$ per cent better or worse than average obeyed a probabilistic dynamic, as shown in Figure 4. This mathematics can be used to describe the growth or decline of an 'event' that occurs suddenly in a system. The important lesson of the probabilistic mathematics of the event dynamics is that even if we knew for certain that a given event actually had properties that increased its probability of growth and decreased its probability of decline, the resulting growth or decline observed would still be fairly uncertain - being as the sloping curves in Figure 4 testify. Instead of a 'step function' clearly marking the growth to maximum value of a 'better' than average event and the immediate collapse of a 'worse' than average events, 


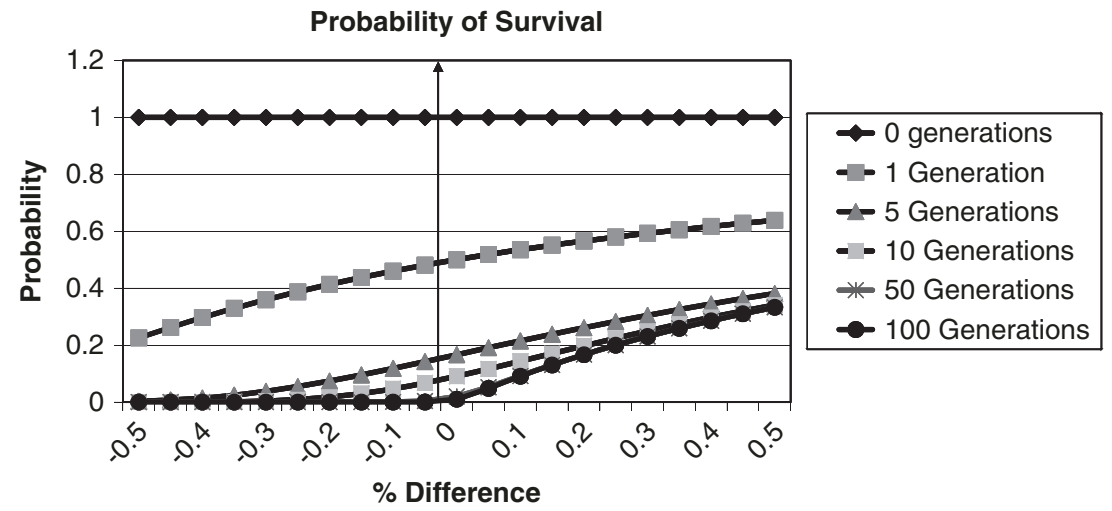

Figure 4: The gradual change in the survival of events with different characteristics.

with zero to the left and 1 to the right of the $y$-axis, we see that survival over time is much more subtle.

Events involving elements with poorer performance than average can still hang around for quite a time, growing to quite a size before regressing, while new behaviour with strong growth characteristics can sometimes regress completely. Luck plays a role in history. Of course, over longer times only events with above average growth characteristics will be permanently retained in the system, but many of these will be lost. Having considered what happens to 'events' once they have happened, we need to turn to the probability that an event of a given type will occur in the first place. The most natural assumption is that individual events are generated according to some 'gaussian spread' around an average. The actual course of events is therefore generated by the combination of the probability of occurrence and survival of different events, and this is shown in Figure 5.

The real question to be answered is about the resilience of a system to novel events, which may have diverse new dimensions and characteristics. The spread of a new virus will start off as a very small, local event, but what matters is how the system responds to it. What is needed is a multi-level 'immune system' and not just one at the level of the individual but also at different organizational levels. We need to use cognitive power, reflection and modelling to try to explore possible novel events, and to see how we may best prepare to deal with them. This will never stop all evolutionary change, but can mitigate and even avoid some catastrophic events that would otherwise occur. Climate change is an ideal example. By considering it and trying to model the system we can explore the probability of various events occurring and how they might unfold. The models are not 'predictive' in the traditional scientific sense, but allow some exploration of possible futures. The more credible these reflections 


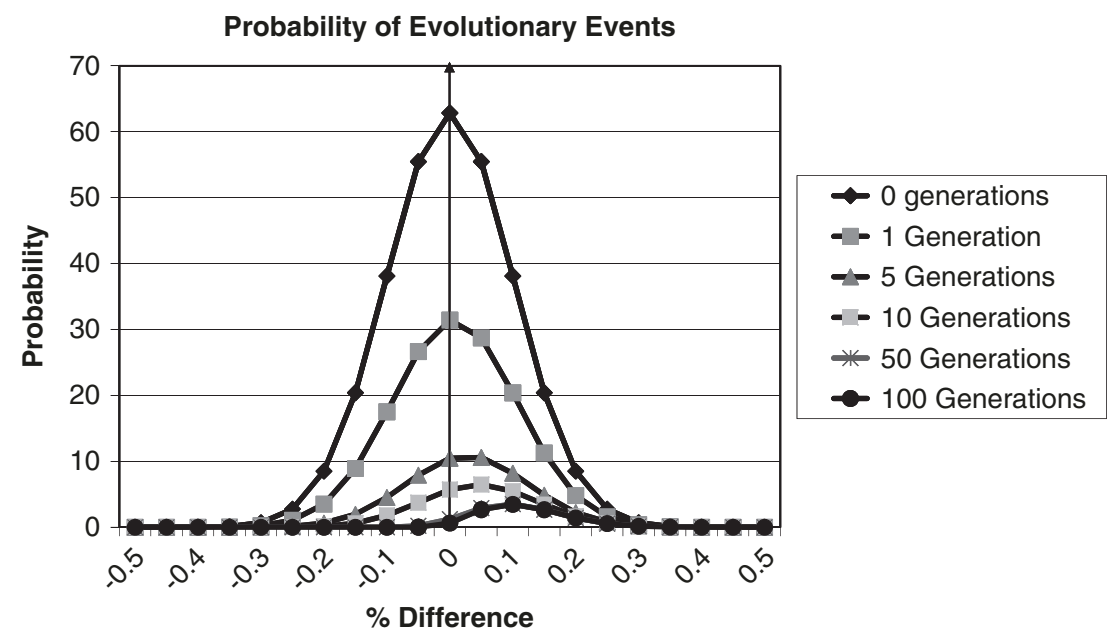

Figure 5: The probability of occurrence and survival of events of different kinds.

become, the more that preparation and mitigation will occur. In the next sections we consider particular examples where reflection, modelling and exploration can lead to successful evolution - systemic structural change - first in the case of the management of major road accidents, and second in the evolution of an aerospace supply chain and of the changing 'performance' criteria that have to be applied to it.

\section{The Complexity of Motorway Accidents}

A recent paper (Butler and Allen, 2008) examined the problem of how best to deal with traffic accidents on the UK motorway network. In this case the 'event' is a major incident, where there is involvement of the emergency services as well as a host of other people concerned with the removal of obstructions, the repair of the infrastructure, the handling of traffic and the practical decisions required to restore the system to full, effective functioning. In order to deal with incidents as smoothly as possible, the series of tasks required need to be established and prioritized, and undertaken in the 'best' order possible, with a minimum of interference between them. The problem that arises, therefore, is that the precise details of every event have to be discovered in real time, and which steps are required and in what order has to be decided. On the whole, the handling of such events had been developed in an ad hoc manner through the collaboration of police, emergency services, and so on.

The Motorists' Forum became concerned about the level of congestion caused by incidents. They commissioned the Highways Agency to carry out a study to see whether it was possible to reduce the time taken to clear incidents, with the 
suggestion that a performance indicator be agreed to halve by 2004 the average time taken to clear the effects of serious incidents. The study was commissioned in 2001. The report found that there had been little prior understanding of the extent of involvement of agencies involved in clearing up after incidents, and that most incidents are minor and require police attendance only. Fire and rescue services (FRS) attended 3 per cent of all incidents, and ambulances somewhat less. In one county, this could equate to less than 10 road traffic incidents per month for the FRS. For these major events, there was little perception and recognition of the range of participants involved in clearing up after accidents. These were the police; the Fire and Rescue services; ambulance and paramedic services; transportation agencies that maintain the road network and their maintenance agents and contractors; hazardous material cleanup services and environmental protection agencies; private towing and rescue companies; and information and private traveller information providers, which include public agencies and private companies that collect, process and disseminate traffic and transport-related information to benefit travellers.

The study brought into sharp focus the differences in work and culture between the different organizations. In particular, some organizations operate around-the-clock and are response-orientated while others are not. Police officers are trained to assume command and make unilateral decisions, whereas fire and rescue personnel act in teams. Private sector organizations, such as towing companies, are profit-driven and therefore mindful of the amount of time they are involved and the resources they apply. Media often view an incident primarily for its relative newsworthiness and may not give adequate consideration to additional information from which the travellers could benefit.

Most importantly, no single organization was statutorily responsible for incident management in a holistic sense although the police enjoyed primacy at the scene.

The report identified a number of key issues:

1. There was a lack of definition about roles and responsibilities of participants and no national guidelines or procedures.

2. Inadequate training of site management.

3. Difficulties in pinpointing the location of an incident, particularly when they are reported via personal mobile cell phones. These are not linked to any location referencing systems (unlike emergency roadside telephones) that the emergency services are able to access.

4. Many of the organizations involved in incident clearance do not follow the same boundaries as the emergency services, which themselves may follow slightly different boundaries or cover more than one county.

5. The emergency services can access the site by using the hard shoulder to reach the scene of an incident or try to part the traffic in the middle of the 
running lanes. Non-emergency services do not carry blue lights on their vehicles and frequently have to 'battle' through the tailback to get to the incident.

6. At the scene, the senior police officer present was accepted as 'in the lead' but this was not always clear to the non-emergency services.

7. Time taken to plan and set up diversion routes.

8. Difficulties with vehicle recovery such as having the right equipment, needing to close the opposite carriageway and the work being unpaid.

9. Provision of accurate and timely information to motorists was considered a low priority.

A major part of the report was a detailed task analysis to gain a clearer understanding as to what actually goes on 'on the ground' during the unfolding of an incident. This helped inform the development of new policy designed to respond to what had been revealed, and thereby to improve the overall processes required to deal as smoothly as possible with a severe incident.

Two types of roles were involved in dealing with incidents. The first were the specialist roles filled by the FRS, ambulance, recovery, road maintenance and the second role is that of the de facto manager. The latter role was normally filled by a police officer who took overall responsibility for the situation. The specialist roles are convergent and involve goal-oriented, highly focussed work with a clear assessment - action - completion format where good performance is easy to assess. The 'incident manager' role is divergent, however, and involves creating an ongoing, transient organizational infrastructure in which the other specialists (which may well include the police in other roles) undertake their work.

The key cognitive skill of the incident manager was to build and maintain an accurate mental model of the incident so that appropriate situational knowledge and knowledge from other domains and constraints can be integrated into the decision-making and planning process. Other key cognitive skills were the ability to scavenge for information repeatedly and proactively; to maintain and test the veracity of the models; to cope with and reduce areas of uncertainty; to deal with multiple streams of information; to prioritize and to rapidly construct and modify short- and medium-term action plans on an ongoing basis. In large incidents, this was a considerable mental activity and one that is not wholly apparent to the observer but nonetheless critical for that.

Responsibility for the management of incidents was distributed over time, over different people and different organizations. Although traffic police normally arrived first on motorways and trunk roads, in rural areas the FRS (possibly retained) could be first to the scene as they may be section officers in urban areas. The control room function also has some form of incident management responsibility. Control of complex incidents was handed over to a senior sergeant sooner rather than later. In addition, more senior officers 
may come out to large incidents. Functional liaison occurred at task level, supervisory level and management level. The mantle of control was distributed and mobile. The need to collect evidence for a later court hearing added to any clearance time. Detailed area searches can be time-consuming but are not common.

The analysis divided the process of clearing up incidents into 26 main tasks. The analysis converged in three types of category:

1. Information processing - passing and processing information around the system.

2. Action management - normal management functions, such as planning, monitoring and checking, evaluating, prioritizing, planned allocation of priorities, coping with undetected circumstances, forming a conceptual model of what is going on.

3. Action tasks - causing action to be taken and may be expected to result from action planning and communication inputs.

Of 304 individual subtasks identified, 179 (59 per cent) involved action management, 53 (17 per cent) were information processing tasks and 72 (24 per cent) were action tasks. This was probably the most significant finding from the study. Evidence from interviews and workshops suggested that preparation and training for incident management concentrated on command structures and completion of the specified activities, whereas information processing tasks were lumped together under a general and unexpanded heading of 'communications' and largely invisible management activities that made up 59 per cent of tasks were largely ignored.

This finding explains an observed disparity between traffic officers undertaking the work and the world of operational analysis, which failed to give due importance to the cognitive skills and operational competencies required. The traffic officers were left with the correct perception that they are undertaking a skilled and specialist task that seems unmentioned and acknowledged by the analysts. This gap maps onto the cognitive aspects of the tasks that form a significant portion of what the person managing the incident actually does. In other words, the 'task analysis' provided a simple form of complex systems, multi-agent modelling, as it examined what happens through the eyes and cognitive processes of the different people involved, rather than assuming some mechanistic picture of a system operating smoothly to accomplish a given overall task - with some underpinning assumption of perfect knowledge and analysis. In reality, incidents vary considerably in their precise details, the different services arrive in an unpredictable order, and there are ongoing particularities of the situation that need to be assessed and analysed, as well as a coordination problem that is fluid, unique in details requires real-time responses. This is shown in Figure 6. 


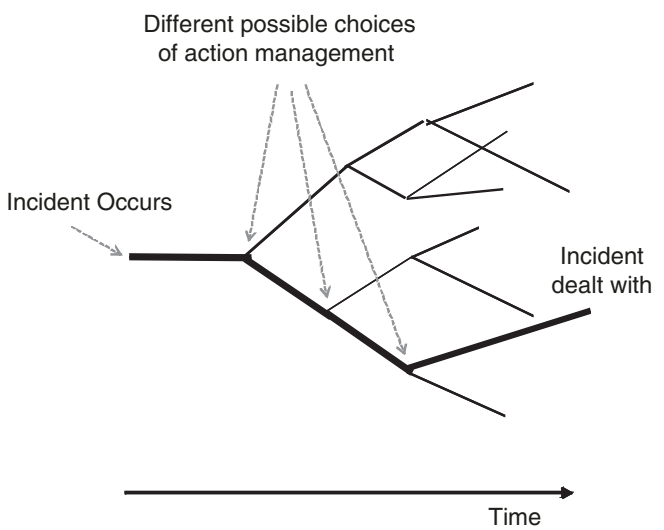

Figure 6: The choices and decisions made in real time in dealing with any particular incident have a tree of possible outcomes. Only one path will be followed in any single incident.

This is typical of complex systems approaches to reality, that is, to situations in which fluctuations, structural change and interpretive frameworks are all evolving together in real time, requiring a degree of creativity and awareness that cannot be encapsulated in any formal set of fixed rules (Allen and Varga, 2006). The 'task analysis' provides us with a simple categorization of the structure of event management in this case and separates out the 'action management tasks' (59 per cent) involving the weighing up of different factors and the matching with possible actions, from the simple information processing (17 per cent) and actual actions (24 per cent). In other words, it focuses on the complex decision-making processes that are required in dealing with an incident.

Clearly, in order to improve event management, those who manage incidents need to be properly trained in undertaking cognitive, action management types of activity, including not only basic cognitive competencies, but also an awareness of errors, faults, risks and how they may occur and how they can be managed, so they can be best avoided. This was absent from any formal training scheme. This aspect was not recognized and not valued except by the actual practitioners. Incident management is unlikely to be improved unless we explicitly address 59 per cent of the tasks.

This shows us that these traffic events are complex systems, in that in each particular instance each choice and particular sequence of responses will produce its own characteristic outcome, and each incident is likely to be different from the preceding one. Therefore, obviously, there is a difficulty in learning how to respond, as the degree of novelty will not be evident straightaway and the lesson of previous incidents is only partial. In effect, long and varied experience may help, but on the whole there is a serious need to prepare those involved in making these choices with the nature of this kind of situation. The unfolding of a particular incident and its handling are complex systems 
because they show non-linear interactions of the parts - the precise sequence and size of each initiative affects the path of the system, and this is because the process is not a sequence of 'equilibria' that can be made in any order. Instead, the overall event is a non-equilibrium phenomenon where the situation is altered as it goes along, with the decisions and qualities of the interventions (Allen, 2001a, b).

In order to deal with these issues, the report recommended that the overall responsibility for incidents should pass from the police to the Highways Agency. This was so radical that a review was commissioned. The review found that traffic police spent only 5 per cent of their time on crime-related tasks and the majority of their time on those that could be undertaken by other organizations. It also highlighted the strategic importance of the trunk road network, which carries 36 per cent of all vehicle kilometres travelled and on which congestion was estimated to cost the UK economy $£ 2.6$ billion annually and the resources allocated for incident management were estimated to be only $£ 86$ million annually.

To deliver improvements, three main changes were required:

- The introduction of seven new regional control centres, jointly staffed by the Highways Agency (HA) and police, to manage resources on the trunk road and coordinate incident supervision.

- The introduction of 'Highways Agency Traffic Officers'. These uniformed HA staff would patrol the trunk road network and deal with many non-core activities currently undertaken by police.

- A national enhancement to the existing contractor provided Incident Support Units to ensure a consistent service, response times and livery.

A single, national set of standards and protocols for incident management, control and monitoring for the trunk road network, agreed upon with the police, was also identified as critical to the safe running of transferred services.

The Traffic Management Bill was introduced on 11 December 2003 and published on 12 December 2003. The second reading on Monday, 5 January was announced almost immediately after its introduction, the third reading was on 16 March 2004 and Royal Assent was received on 22 July 2004.

An important feature of this case study is that it demonstrates the multiple layers on which complexity emerges and affects society. The better management of 'accident events' on UK roads required the recognition that each incident needed an element of adaptive learning because the details of the particular incident and the choices and responses that had to be made led to multiple possible pathways of the 'incident system' into the future. The recommendations aimed at improving the institutional framework and training in order to allow improved learning to occur. However, the legislation itself also went through an unpredictable path of successive incidents and events in which either it 
would have been modified considerably in its eventual form, or it might have failed to pass at all. Clearly, both the matter being legislated and the legislation process itself were examples of complex systems, with different possible outcomes, with low predictability but significant, structural and qualitative differences in the result.

\section{Organizational Evolution}

The case described above focuses our attention on 'negative' events such as road accidents. But it is important to realize that the same principles apply for 'positive' events - such as those that are involved in the evolution and development of people, economies and societies. The idea is based on organizational cladistics (McKelvey, 1982, 1994; MCCarthy et al, 1997). For example, the organizational evolution of aircraft manufacture has been important for the growth and success of the whole world economy, both for business and for pleasure. In this example we can consider production innovations such as new techniques and working practices and see how their appearance and survival has been of great importance to the industry. Furthermore, these innovations are really just examples of potentially positive 'events' that need to be tested out within firms in order to see whether they are improvements.

Large commercial aircraft manufacturers traditionally defined and specified exactly what their first-tier suppliers should produce for them. This practice that expressed a high level of buyer dominance over suppliers has gradually changed as the result of a worldwide reorganization of the industry. Aerospace supply chains have evolved qualitatively as different practices and conventions have emerged that profoundly shift the relationships between the participating companies (Giunta, 2000). Recent developments in supply chain strategies are therefore based on a focus upon the sharing of skills and capabilities (Cousins and Spekman, 2003), and in the perspective of creating technologically more advanced products, supply chains have become learning communities with skills in creating and transforming knowledge (Preiss and Murray, 2005). In that sense, Easterby-Smith et al (2005) highlight the absorptive capacity of a firm to appreciate and process external knowledge and the learnings from past experiences. Engeström (1987), however, is more concerned about the learning and development experienced when moving away from the old. And this is about the creation of the new, that is, innovation.

Risk-sharing partnerships combine these views in practice by bringing together the best expertise available to produce totally new concepts of aircraft design. These partnerships are the response to the need for sharing of skills, expertise and capabilities. This is driven by the fact that an airframe manufacturer cannot carry such huge investments in capital and expertise alone. The tight integration of risk-sharing partnerships can facilitate a totally 
new aircraft concept (Rose-Anderssen et al, 2008). Here we briefly describe the emergence and development of practices, techniques and characteristics that underlie the capabilities of modern aircraft manufacturers to produce higher-performing, and complex products.

The major civil aviation manufacturers had five basic dimensions of performance:

\section{Quality}

2. Cost efficiency

3. Reliable delivery

4. Innovation and technology

5. Vision

The stage in the life cycle of the product, or the market situation, determines what mix of these is required as the platform or product moves from design and conception, through initial prototyping and production to an eventual lean production phase. In order to attain these performances, 27 key characteristics or practices were identified within supply chain relationships. A questionnaire was formulated to enquire into the opinion of important individuals within these key aerospace supply chains in order to understand better the underlying beliefs that affect the decisions with regard to the structure of supply chains.

The first three dimensions of performance can be seen as those of 'operationality' - the successful functioning of a production system in producing high quality products reliably and cost effectively. The dimensions 4 and 5 , however, are really about the supply chain future. They are about the creation of the next product that will become the focus of operational behaviour later. Thus, these two dimensions involve the creative, exploratory behaviour without which there will be no long-term future.

The practices that are considered are:

1. Outsourcing competitive advantage

2. Outsourcing what is easily imitated

3. High level of collaborative relationship

4. Arm's-length relationship

5. Long-term relationship

6. Formal partnership

7. Subcontracting whole systems and sections

8. Flexibility of operations

9. Risk-sharing

10. Sharing knowledge

11. Offsets as part of sales contract

12. Culture of continuous improvement

13. Ability to handle cultural differences 
14. High level of dominance over supplier

15. High level of planning and control

16. Easy dialogue with supplier

17. IT system integration

18. High levels of integration in chain

19. Responsive to market change

20. Transparent organization

21. TQM procedures

22. Just-in-time delivery

23. Lean practices

24. Explorative learning practices

25. Investment in training

26. Supplier development

27. Monitoring Suppliers

Without going too much into the details of the study, managers were asked to fill in their views on the importance of the 27 practices in helping the five difference performance criteria (Cost, Quality, Reliability, Innovation, Vision) and also in estimating the degree of conflict or synergy between each pair of practices.

This reveals that, on average, managers looking for quality as the overriding quality of their output would pick practices 7, 10, 12, 21 and 26. If Cost efficiency is the dominant consideration then they would pick7, 12, 21 and 26. For Delivery reliability: 7, 8, 12, 15, 18 and 22. For innovation and technology the practices would be $1,3,7,9$ and 10, while for Vision there is a weaker response, and only practices 3,9 and 10 are chosen. This gives us a first estimation of how practices can be chosen in order to achieve a desired supply chain performance. Clearly, for sustainable behaviour of the firm, however, we may want to have a cost-efficient 'cash cow' element running a mature product production but also have a 'design team' part that has the visionary and innovative practices that will be needed to define a future 'cash cow'. Therefore, one should envisage aerospace relationships to encompass both lean production elements and also visionary, design relationships that are used to define new platforms and products.

However, the real issue is that managers could only fill in the questionnaires sensibly if they had already learned from experience about the effects of practices and their combinations. In reality then, each process innovation is an 'experiment' in which a practice only takes off if it is believed that it is improving performance in the necessary way, and therefore what we have is an evolutionary process driving organizational change over time. We therefore developed a 'learning' model in which random practices were launched successively, and the model shows which will actually 'take off', using the responses of managers to determine the parameters governing the performance and interaction of 
practices. Each practice is supposed to have a cost in implementation, and therefore following the introduction of a practice if the performance does not increase more than the cost of its introduction then it is considered a failure and is withdrawn.

In this way, agents attempt to introduce successive practices in random sequences, and some, by luck, increase the performance of the supply chain much faster than others. The performance is measured by a weighted sum of the five criteria mentioned above. The model proceeds by calculating the performance (in terms of the weighted criteria) for the bundle of practices initially present and adds a random practice. If the gain in performance is less than the cost of the implementation, then the practice is removed again. In this way, overall performance increases in steps given by the matrices of performance, depending on the particular sequence that occurs.

The model represents the dialogue between the process 'inside' an organization or supply chain and the demands placed on it by the outside world. Firms proceed by 'trial and error', as the collective interactions are difficult to anticipate, and therefore luck leads to different firms learning which bundles of practice lead to what performance at different rates. For example, if we require high-quality production. We find Figure 7.

This shows us the 'clusters' of practice that generate particular types of performance, and also how much they gain from the synergy within the cluster. The program can now be run with the weights now chosen to maximize successively the different dimensions of performance. The practices retained for cost efficiency, reliable delivery, innovation and vision are shown in Figure 8.

The effects of the patterns of synergy and conflict are strong, and therefore the results coming from the retention of particular patterns are not necessarily obvious. Knowledge of the effects of interaction can therefore be of considerable advantage in creating a successful supply chain. This shows the importance of considering the systemic, collective effects of any organization or supply chain and also why each acquisition of a practice is really an 'extreme event' because it is one that changes the firm, and eventually the whole industry. Learning and

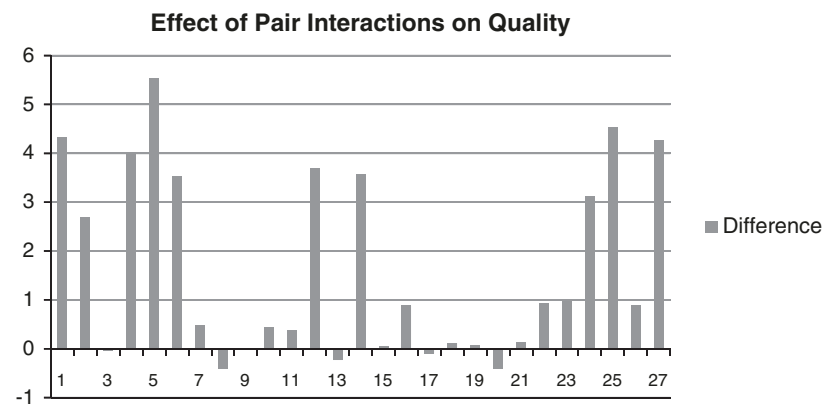

Figure 7: Practices that lead to high quality production. 


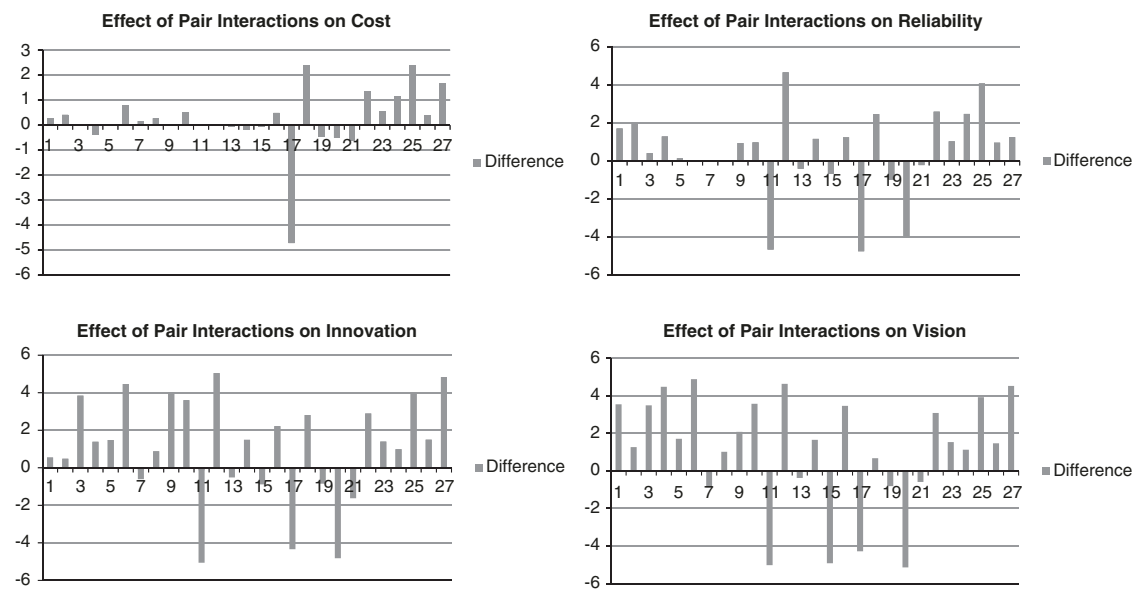

Figure 8: The different practices retained for the different dimensions of performance.

evolutionary changes are all marked by the occurrence of 'extreme events' that make the future qualitatively different from the past.

\section{Discussion}

In this article we have presented a discussion of 'extreme events' and used three different examples to illustrate the link with complexity and evolution. In the first, we considered the mathematics that underlies much of the discussion of extreme events of a particular type within a qualitatively fixed context. Power law probability distributions and rank-size rules are what emerge as governing the probability (future and past) of the size and frequency of events. For earthquakes, storms and epidemics the focus is on negative events, while for city and firm sizes it is generally viewed as positive. However, all of this ignores any qualitative evolution that might underlie these systems. In reality, cities may grow because they become the focus of expertise and knowledge of some particular kind, and this both fits them into the larger structure of the urban hierarchy and shapes the employment and skills structure of its inhabitants. Similarly with firms, it seems absurd to consider firms as differing only in the amount of good and bad 'luck' that they have. In reality, it is their particular differences and dimensional diversity that will matter, though 'luck' will also play a role. As soon as we include even the idea that events and novel entities may have some intrinsic qualities that bias them towards stronger or weaker growth within the complex, multi-dimensional web of interactions that they encounter, then we see the simplistic nature of the simple 'stationary' power law assumptions that ignore evolution and change. In fact, the precise shape of the evolving probability distribution is really a function of the non-linear 
interaction parameters and these are changing as behavioural exploration and differential retention change the nature of the system over time.

In the road accident example, we show that these constitute a series of extreme events that have individual features that make their handling a matter of real-time learning. The analysis of the previous ad hoc management procedures revealed a number of serious problems, particularly as regards the cognitive issues involved in deciding the nature, degree and requirements of the incident. This also illustrates the important point that simply letting the system continue does not necessarily lead to successful 'self-organizing' processes, because in such systems the structure and interactions within it mean that it not possible for participating agents to learn how to make the system work better. A study led to the framing of a policy document that became law, itself a particular type of extreme event, changing fairly radically the way that such events were handled. Although there has not yet been a definitive study of the overall outcome of the new policy, the recognition of the issue of 'decisionmaking' in real time, under uncertainty, has led to modified and improved training for the emergency staff and this does seem to have been considered a success.

The examples of organizational evolution of the supply chain are really about the difficulty in knowing how performance will be affected in reality, if some new practice is introduced in an organization or supply chain. The approach coming from complexity modelling shows us that the way forward is to try to learn by experiment, with systematic exploration and monitoring of the outcomes.

The method that we have developed is in fact a completely general approach to complex systems. The 'event' here is in terms of a 'practice' - a particular technique, activity or action that it is initially believed will enhance the collective performance of the system. The model allows us to examine how different practices will contribute to, or detract from, different possible dimensions of performance that the overall production system requires in order to succeed with its customers or in the marketplace.

Successful management must behave as evolution does and make sure that mechanisms of exploration and experiment are present in the organization. Discussions of 'extreme events' in terms of a fixed power law distribution are really not about learning, or management, as if the distribution is assumed 'fixed' then it is also assumed that nothing can be done about it. Thus it may be correct for earthquakes, storms and epidemics, as the occurrence of these events are difficult to influence and all the effort goes simply into the management of their effects. This is useful, but of course the real world is also about evolutionary, qualitative change whereby the nature of threats and opportunities actually changes over time. In these situations creative destruction is one way of describing what occurs and so it may be important to recognize that learning involves short-term risk-taking, while not-learning involves longer-term extinction. We see that the appearance of some predator may be 
'catastrophic and extreme' for the local prey, but this leads to evolutionary selection that eventually deals with the change. Micro-diversity is therefore the important mechanism by which natural evolution occurs and that creates resilience. In human systems this involves not only genetic and cultural factors, but also technological and infrastructural micro-diversity. Clearly, economic 'optimality' is a certain driver for unsustainable systems, as the optimality is judged within some relatively short time-scale. What is required is continuous modelling and experimentation so that potential threats are constantly explored, understanding updated and emergency procedures tested, both through simulation and wherever possible in real trials.

Complex systems' thinking tells us that we are forever at risk - evolving into an unknown future, with sometimes interesting, sometimes painful consequences. Though we construct edifices of routine and regularity into our system, both the need to innovate and change and the occurrence of some unexpected environmental change will always assert themselves at some point in time, and we shall be forced to move to a new, temporary set of routines and regularities. Rational thinking has revealed the limits to rational thinking and we see that evolution springs from exploration both within the environment and within ourselves, and these ongoing processes both create and allow uncertainty. Uncertainty and surprise are a necessary feature of life itself and we need to prepare as much as possible for the bad events without making our system so rigid that nothing good ever happens either. Recent events in financial markets illustrate this idea. It is naive to think that systems will automatically evolve to optimal solutions and that we can simply suppose that market mechanisms or unguided self-organization will necessarily lead to successful and stable systems. Instead, we need always to doubt our beliefs and the current system, and use models to explore possible instabilities, and different organizational structures according to our current understanding, always keeping in mind that although experience and models are all we have, reality will always go beyond them, and give us an inevitable surprise.

\section{Acknowledgements}

This work was supported by the ESRC RES-000-23-0845, Aerospace project with Sheffield University and also by the EU Qoscosgrid Project: FP6 STREP 033883.

\section{References}

Allen, P.M. (1976) Evolution, population dynamics and stability. Proceedings of the National Academy of Science, USA 73(3): 665-668.

Allen, P.M. (2001a) Knowledge, ignorance and the evolution of complex systems. In: J. Foster and S. Metcalfe (eds.) Frontiers of Evolutionary Economics: Competition, Self-Organisation and Innovation Policy. Cheltenham, UK: Edward Elgar. 
Allen, P.M. (2001b) A complex systems approach to learning, adaptive networks. International Journal of Innovation Management 5(2): 149-180.

Allen, P.M. and Ebeling, W. (1983) Evolution and the stochastic description of simple ecosystems. Biosystems 16: 113-126.

Allen, P.M. and Varga, L. (2006) Complexity: The co-evolution of epistemology, axiology and ontology. Nonlinear Dynamics, Psychology, and Life Sciences 11(1): 19-50.

Butler, M. and Allen, P. (2008) Understanding policy implementation processes as selforganizing systems. Public Management Review 10(3): 421-440.

Cousins, P.D. and Spekman, R. (2003) Strategic supply and the management of inter- and intra- organizational relationships. Journal of Purchasing and Supply Management 9(1): 19-29.

Easterby-Smith, M., Graca, M., Antonalopoulou, E. and Ferdinand, J. (2005) Absorptive Capacity: Tales from the Field. EBK Publications, Working Paper 2005/08 ESRC.

Engeström, Y. (1987) Learning by Expanding: An Activity-theoretical Approach to Developmental Research. Helsinki, Orienta-Konsultit.

Giunta, A. (2000) Large firms and subcontracting relations in commercial aircraft industry. In: A. Giunta, A. Lagendijk and A. Pike (eds.) Restructuring Industry and Territory: The Experience of Europe's Regions. Norwich, UK: The Stationery Office, pp. 37-56.

McCarthy, I., Leseure, M., Ridgeway, K. and Fieller, N. (1997) Building a manufacturing cladogram. International Journal of Technology Management 13(3): 2269-2296.

McKelvey, B. (1982) Organizational Systematics. California: University of California Press.

McKelvey, B. (1994) Evolution and organizational science. In: J. Baum and J. Singh (eds.) Evolutionary Dynamics of Organizations. Oxford: Oxford University press, pp. 314-326.

Preiss, K.J. and Murray, P.A. (2005) Fashions of learning: Improving supply chain relationships. Supply Chain Management, Emerald 10(1): 18-25.

Rose-Anderssen, C., Ridgway, K., Baldwin, J.S., Allen, P., Varga, L. and Strathern, M. (2008) The evolution of commercial aerospace supply chains and the facilitation of innovation. International Journal of Electronic Consumer Relationship Management 2(1): 63-84. 